\title{
Metabolic syndrome and liver-related events: a systematic review and meta- analysis
}

Huina Ren, Junna Wang, Yue Gao, Fuwei Yang and Wenxiang Huang*

\begin{abstract}
Background: Previous studies have suggested that metabolic syndrome (MetS) and its component conditions are linked to the development of many benign or malignant diseases. Some studies have described relationships among metabolic syndrome or diabetes and liver cancer, but not many articles described the relationships between MetS and cirrhosis, acute hepatic failure, end-stage liver disease, and even death. However, liver cancers, cirrhosis, acute hepatic failure, end-stage liver disease, and liver-related mortality-collectively described as liverrelated events (LREs) - may have different relationships with MetS. We undertook this meta-analysis to examine the association between MetS and LREs, and to determine whether geographic region or hepatitis B virus (HBV) positivity might influence the association.

Methods: Relevant studies were identified from PubMed, EMBASE, and the Cochrane database. Two reviewers independently searched records from January 1980 to December 2017. The search terms included 'metabolic syndrome', 'diabetes mellitus', 'insulin resistance syndrome', and 'metabolic abnormalities', combined with 'cirrhosis', 'hepatic fibrosis ', 'hepatocellular carcinoma', 'complication', 'LRE', 'HCC', 'liver-related events', and 'liver cancer'. No language restriction was applied to the search. We chose the studies reporting an association between MetS and LREs. We used Begg's and Egger's tests and visually examined a funnel plot to assess publication bias. All analyses were conducted in Stata 14.0 software.
\end{abstract}

Results: There were 19 studies (18 cohort and 1 case-control) included in the analysis, with a total of 1,561,457 participants. The subjects' ages ranged from 18 to 84 years. The combined analysis showed an overall $86 \%$ increase risk of LREs in cases with MetS (RR: 1.86,95\% Cl: 1.56-2.23). The funnel plot was asymmetrical, and the Egger's test $p$ values showed a publication bias in this meta analysis. However, through the trim and fill method, we obtained a new RR value for LREs with MetS of 1.49 (95\% Cl: 1.40-1.58, $p=0.000$ ). There was no obvious difference with the two answers, so we concluded that the results were robust. For hepatitis B positive patients, the RR for MetS and LREs was 2.15 (95\% Cl:1.02-4.53, $p=0.038)$, but for the hepatitis B negative patients, the RR was 1.85 (95\% Cl:1.53-2. $24, p=0.000$ ). And for non-Asians, the RR for MetS and LREs was 2.21 ( $95 \% \mathrm{Cl}: 1.66-2.69, p=0.000$ ), while for Asians, the RR was 1.73 ( $95 \% \mathrm{Cl}: 1.35-2.22, p=0.000)$.

Conclusions: This meta-analysis showed that MetS is associated with a moderately increased risk of LRES prevalence. Patients with MetS together with hepatitis B are more likely to develop hepatic events. For non-Asians, MetS is more likely to increase the incidence of LRES.

Keywords: Metabolic syndrome, Diabetes mellitus, Insulin resistance, Metabolic abnormalities, Hepatocellular carcinoma, Cirrhosis, Liver-related events, Meta-analysis

\footnotetext{
* Correspondence: wenxiang_huang@hotmail.com

Department of Infectious Diseases, The First Affiliated Hospital of Chongqing

Medical University, Chongqing 400016, China
}

(c) The Author(s). 2019 Open Access This article is distributed under the terms of the Creative Commons Attribution 4.0 International License (http://creativecommons.org/licenses/by/4.0/), which permits unrestricted use, distribution, and reproduction in any medium, provided you give appropriate credit to the original author(s) and the source, provide a link to the Creative Commons license, and indicate if changes were made. The Creative Commons Public Domain Dedication waiver (http://creativecommons.org/publicdomain/zero/1.0/) applies to the data made available in this article, unless otherwise stated. 


\section{Background}

Because of a significant increase in the incidence and mortality of hepatocellular carcinoma (HCC), this cancer has become one of the most common malignancies and a major cause of death worldwide. In recent years, chronic liver disease has become a major cause of death in the United States, causing a large number of deaths every year, according to national life statistics. All the cirrhotic complications, HCC, and/or liver-related mortality were called LREs. And liver-related death is defined as death related to hepatic events [1]. Viral hepatitis and excessive drinking have been identified as the major risk factors for LREs, but risk factors for approximately 5 to $30 \%$ of HCC cases remain to be identified [2, 3]. Metabolic syndrome (MetS) patients have a high risk of cardiovascular disease. In addition, there is increasing evidence that patients with chronic liver disease are at risk of a higher rate of diagnosis of MetS or diabetes mellitus [4]. Recent studies have shown that MetS might have additional associations with liver disease. Liver cancer is the most severe liver disease. Although MetS is known to promote liver cancer, there is little evidence of whether MetS is associated with cirrhosis, liver failure, liver fibrosis, and death from liver causes.

MetS has become a major public health focus worldwide, and it is related to the occurrence of obesity and the diabetes pandemic. MetS components are a series of risk factors for cardiovascular diseases and it has become an increasingly severe problem globally [5]. The risk factors comprising MetS include obesity, abnormal blood sugar, Increased blood pressure, elevated triglyceride levels, decreased high-density lipoprotein cholesterol levels [6]. Several studies have described the relationship between MetS and diabetes and liver cancer [5], but few have investigated the LREs. The article intended to address this knowledge gap. Recent cohort studies have attempted to further understand the temporal relationship between these factors and to validate previous findings. However, there are few data on the association of LREs and MetS factors, which include obesity, diabetes and MetS.

In this study, we conducted a systematic review and meta-analysis to assess all available evidence to identify an association between MetS and LREs. We additionally analyzed related factors.

\section{Methods}

\section{Search strategy}

We used PubMed, EMBASE, and the Cochrane database for literature retrieval. In December 2017, the following search terms were used without language restrictions: MetS, diabetes mellitus, insulin resistance, metabolic abnormalities, cirrhosis, hepatic fibrosis, hepatocellular carcinoma, complication, LRE, HCC, liver-related event, and liver cancer All references cited in these studies were also reviewed to identify other published articles not indexed in the databases. The systematic review process followed established quality standards for reporting of meta-analyses.

\section{Study eligibility}

The inclusion criteria for studies in the meta-analysis were as follows: either case-control or cohort design; inclusion of subjects over 18 years old; assessment of the effects of MetS on the risk of liver events; and reporting of relative risk (RR) estimates for LREs in subjects with MetS. Only complete papers and published studies in the medical literature were included. Data from summaries, reviews, editorials, case reports, and letters were excluded. Studies reporting no risk ratio (HR) and 95\% confidence intervals (95\% $\mathrm{CI})$, and those with participants with incomplete data were also excluded.

\section{Data extraction}

The following data were collected from each study: first author's surname, type of article, year and country of publication, distribution of age and sex, number and characteristics of the participator, definitions of MetS, risk estimates with corresponding confidence intervals (CIs), and all other information. Quality assessment for cohort studies in this meta-analysis was assessed with the Newcastle Ottawa scale (NOS), as recommended by the Cochrane Non-Randomized Studies Methods Working Group. The scale allocates a number of stars ranging from one to nine.

\section{Statistical analysis}

We used both fixed- and random-effects models to calculate the pooled RR and 95\% CI. We performed an analysis to identify the association between MetS and LREs, and also to analyze any influence of geographic region or HBV positivity. Heterogeneity was assessed with the $I^{2}$ statistic and $p$ value. Funnel plots and Egger and Begg's and Mazumdar's tests were used to assess publication bias. A $p$ value $<0.05$ was considered statistically significant $[7,8]$. The trim and fill method aims to identify and correct the funnel asymmetry caused by publication bias, it can remove a small sample study that causes asymmetry in the funnel plot, and then estimate the center value of the funnel plot with the symmetrical part after trimming. All statistical analysis was performed in Stata 14.0 software.

\section{Results}

Figure 1 details our search steps. A total of 1,561,457 individuals were included in the study. Overall, we 


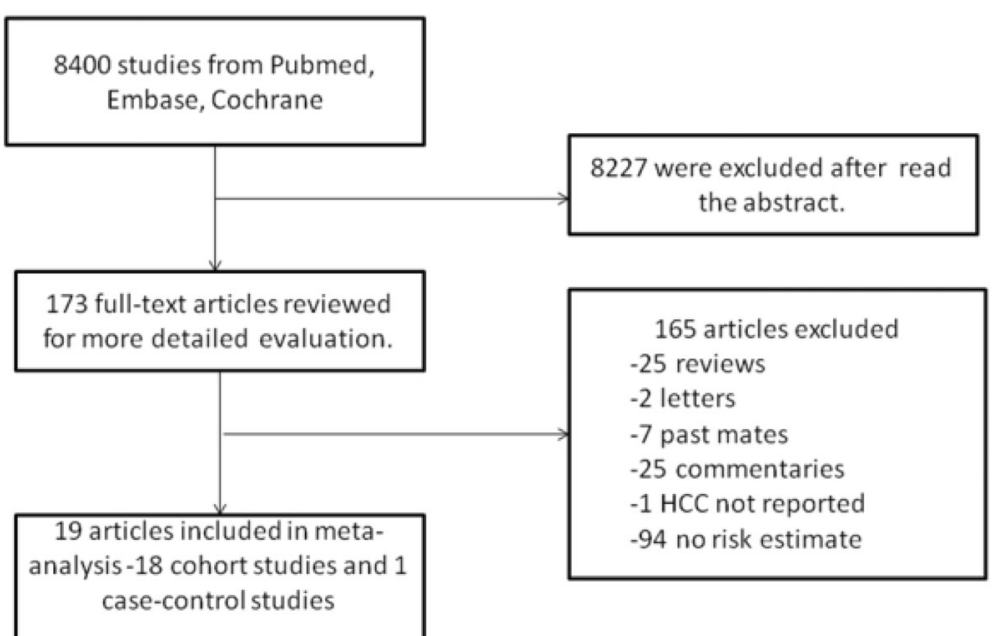

Fig. 1 Flow chart of study selection in this systematic review

identified 8400 studies from different databases, including 4559 studies from PubMed, 173 studies from the Cochrane database, and 3668 studies from EMABASE. After reading the abstract, we initially considered 173 publications to be relevant. Among them, we excluded 25 comments, 2 letters, 7 meta-analysis, 25 comments, 1 unreported HCC, and 94 lacking risk estimates. A total of 19 studies (18 cohort studies and one case-control study) were included in the final analysis [1, 6, 9-25].

\section{Association between metabolic syndrome and liver- related events}

A total of 19 articles were included in this meta analysis. Among the 18 cohort studies, there were 1,546,392 participants, of which 7373 had liver-related events (LREs) (Table 1). The one case-control study was published in 2010 as part of the Third National Health and Nutrition Examination Survey, and it included 2061 liver disease cases and 13,004 controls. The age of participants ranged from 18 to 84 years old. The pooled RR (among 1,561,457 participants from 19 studies) for LREs among subjects with MetS was 1.86 (95\% CI, 1.56-2.23) (Fig. 2).

Because of the heterogeneity of the 19 studies ( $p$ value for heterogeneity $=0.000, \mathrm{I} 2=83.8 \%$ ) (Fig. 2), we used the random-effects model to calculate the combined RR (Fig. 3). On the basis of the random-effects model, no article has a big impact on the results.

\section{Association between metabolic syndrome and hepatocellular carcinoma}

To explore potential sources of heterogeneity, we also performed subgroup analysis for different liver events, such as hepatocellular carcinoma, liver-related death, and cirrhosis.

There were 11 studies about hepatocellular carcinoma, which were cohort studies (Table 1). The RR for hepatocellular carcinoma was 1.76 (95\% CI: $1.33-2.33, p=$ $0.000, \mathrm{I}^{2}=87.6 \%$ ) (Fig. 4).

\section{Association between metabolic syndrome and liver- specific death}

One case-control study and five cohort studies reported risk estimates for liver-specific death (Table 1). The pooled RR for liver-specific deaths was 2.41 (95\% CI: $1.55-3.74, p=0.0005, \mathrm{I}^{2}=86.2 \%$ ) (Fig. 5).

\section{Inclusion characteristics}

We divided the included population into HBV-positive and HBV-negative groups, and then calculated the relationship between MetS and LREs in each group.

In the HBV-positive group, the RR for MetS and LREs was 2.15 (95\% CI: $1.02-4.53, p=0.038, \quad \mathrm{I} 2=64.3 \%)$ (Fig. 6). And in the HBV-negative group, the RR was 1.85 (95\% CI: 1.53-2.24, $p=0.000, \mathrm{I} 2=85.5 \%)$ (Fig. 7).

\section{Terrain analysis}

We divided all the data into Asian and non-Asian regions, and then calculated the relationship between MetS and LREs in each group. We then compared the differences between the groups.

There were 12 articles from Asian countries (Table 1). The RR for MetS and LREs was 1.73 (95\% CI: 1.35-2,22, $\mathrm{p}=0.000, \mathrm{I} 2=85.3 \%)($ Fig. 8$)$. Then there were 7 studies describing non-Asian populations (Table 1). The RR for MetS and LREs was 2.12(95\% CI: $1.66-2.70, p=0.000$, I2 = 76.0\%) (Fig. 9) . 


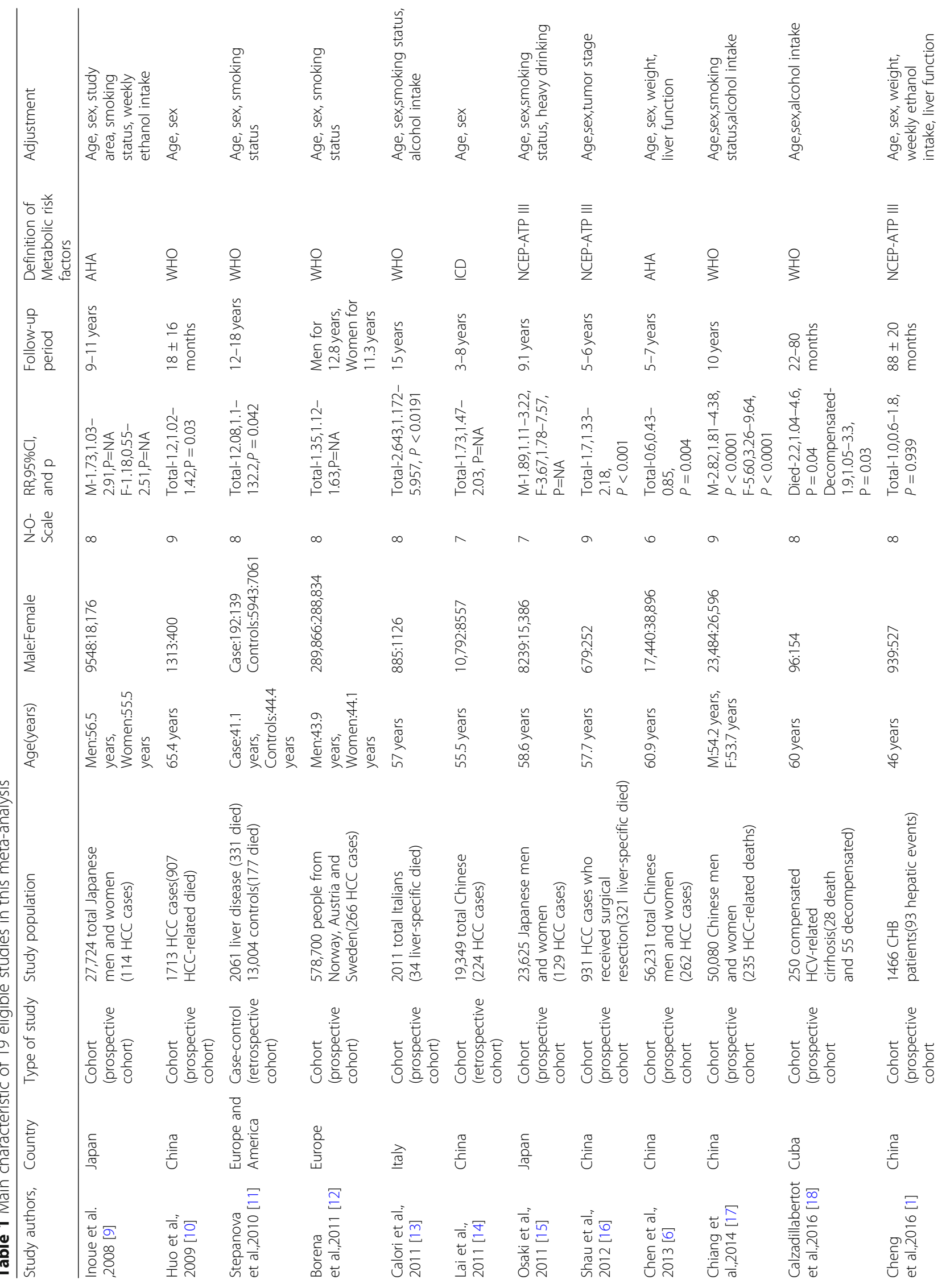




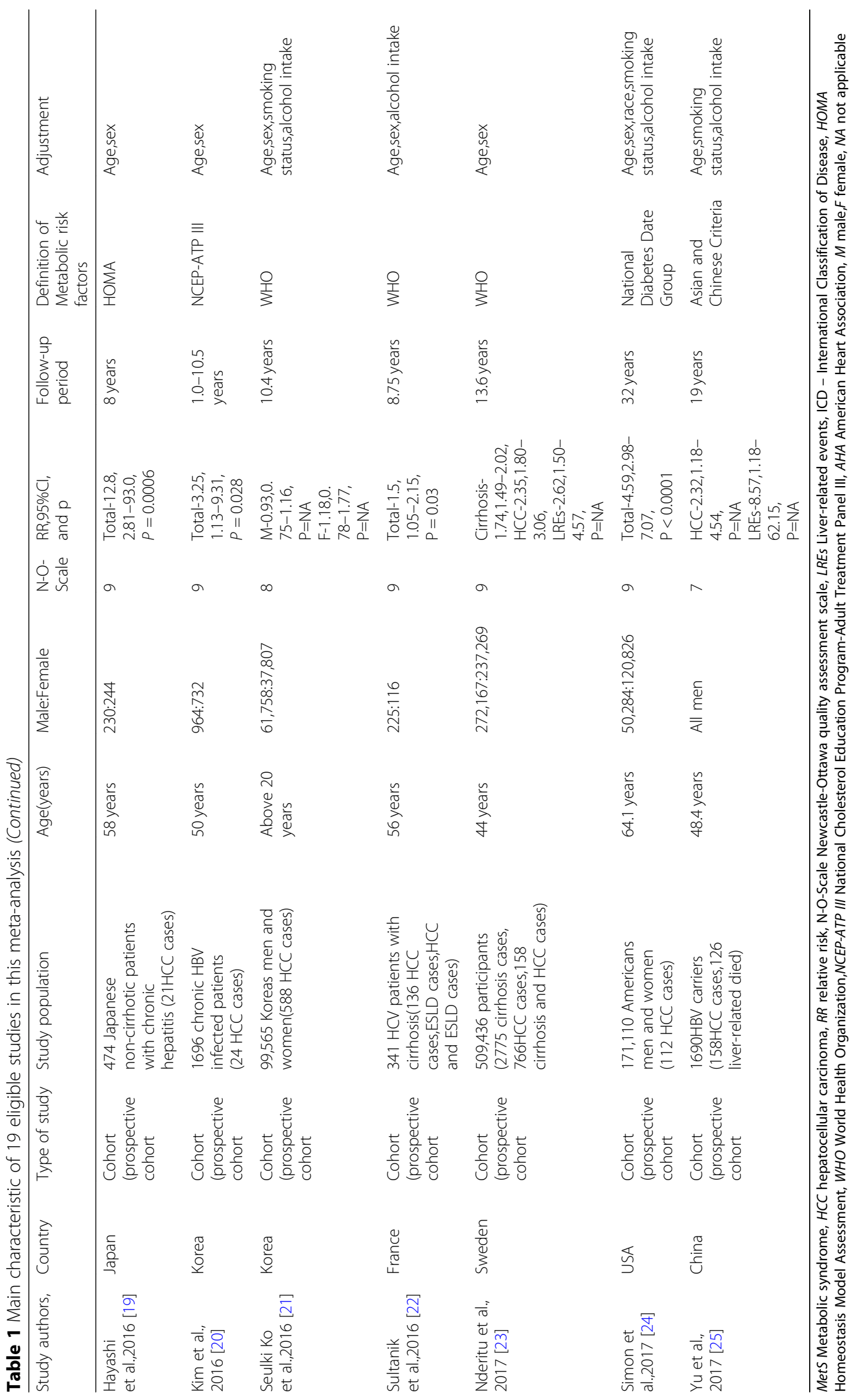




Study
ID
Inoue et al 2008
Inoue et al 2008 S
Huo et al 2009
Stepanova et al 2010
Borena et al 2011
Calori et al 2011
Lai et al 2011 $2019(95 \% \mathrm{Cl})$

Fig. 2 Forest plot: Meta-analysis of the association between metabolic syndrome and liver-related events

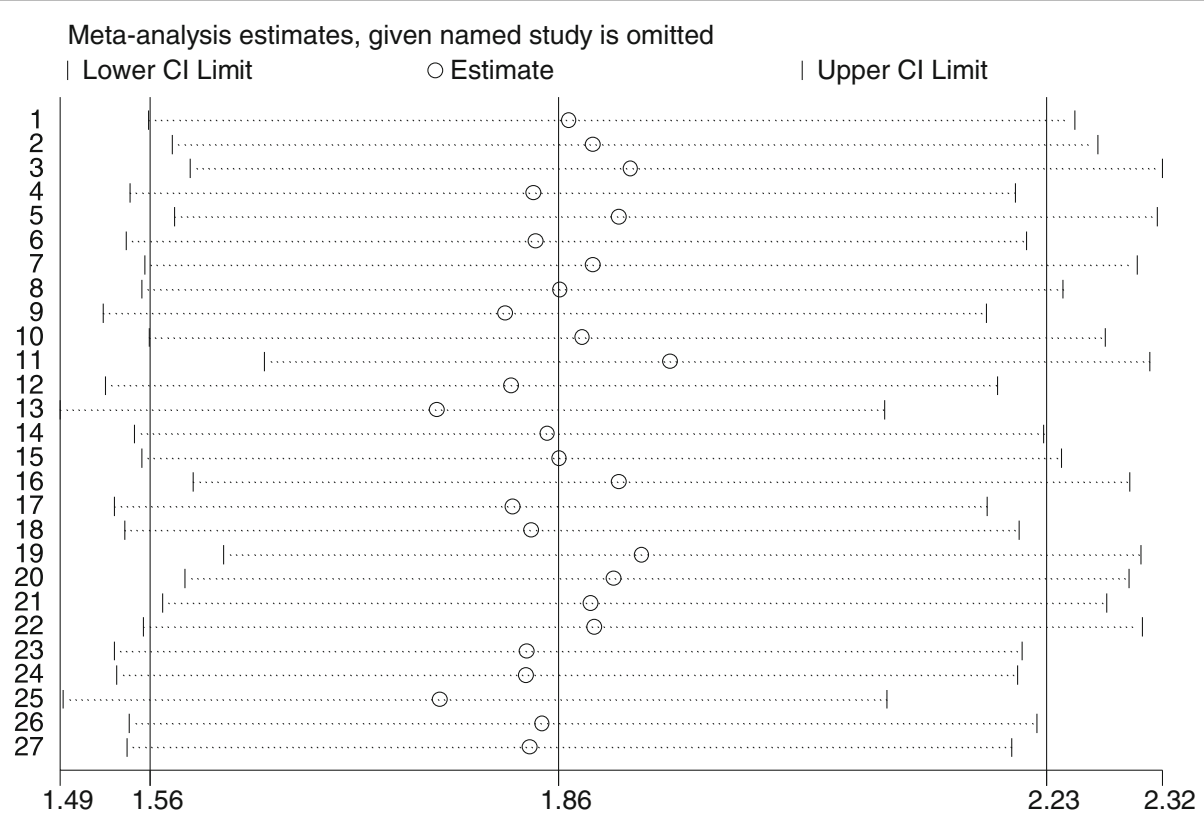

Fig. 3 Meta-analysis random-effects estimates 


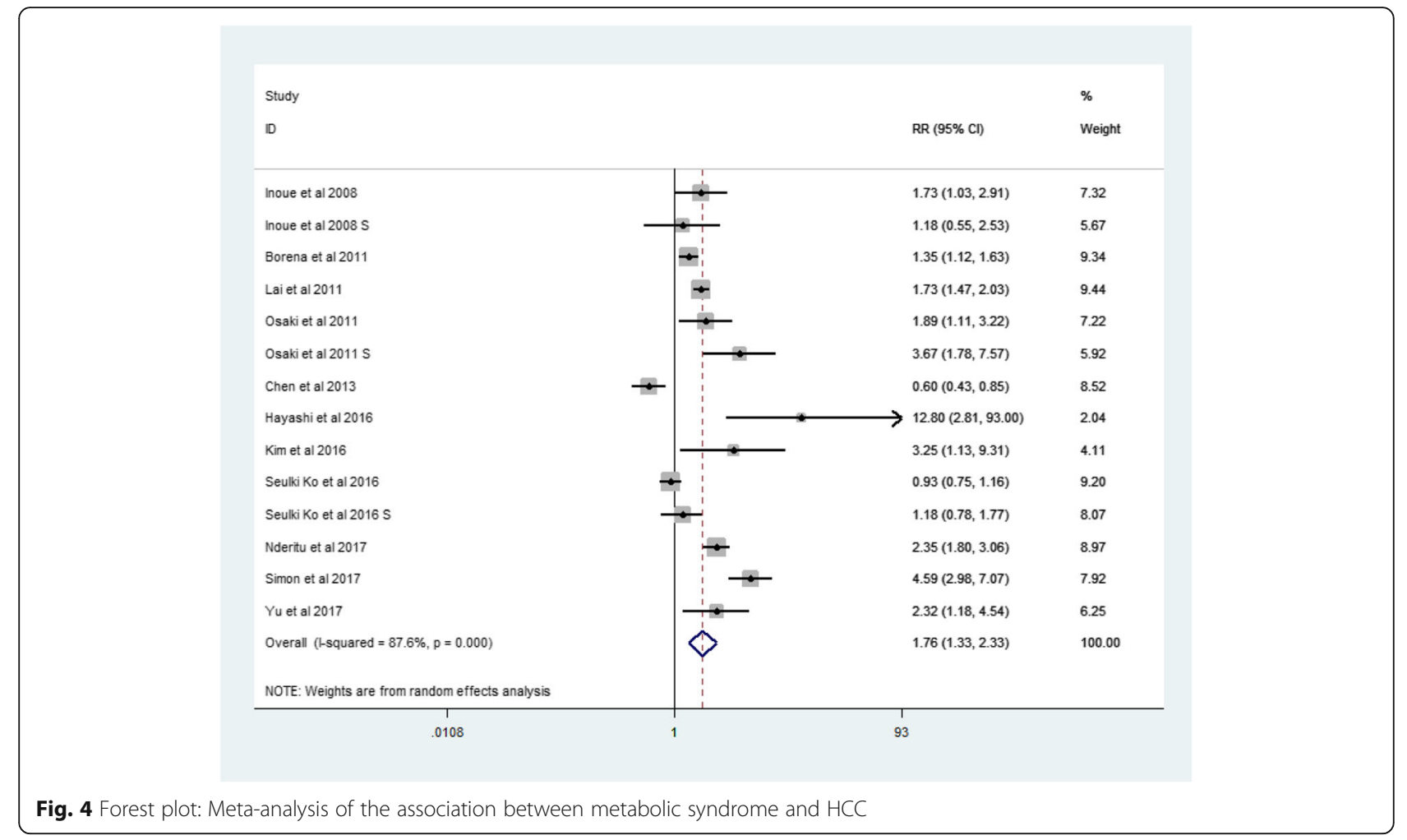

\section{Publication bias}

We used the NOS to assess publication quality and determined credible results with 8.2 stars for the 18 cohort studies and 8 stars for the single case-control study (Table 1). Funnel plots were made (Figs. 10) and Egger's test was performed to assess the publication bias in the meta-analysis. The funnel plot was not completely symmetrical, and the $p$ values of Egger's test were close to 0.05 (Begg and Mazumdar's test: $p=0.08$; Egger's test: $p=0.049)$. Thus, the results suggested that publication bias was present in this meta analysis. Then we use the trim and fill method to correct the funnel asymmetry caused by publication bias, and we get a new RR for MetS and LREs which is $1.49(95 \% \mathrm{CI}: 1.40-1.58, p=0.000)$, and there was no obvious difference compared with our previous results.

\section{Discussion}

In the meta-analysis of 19 studies including 1,561,457 patients with MetS, 16 studies found a statistically significant positive association, while 3 studies showed a negative association. After we removed the negative study indicated by the random-effects model to have affected the results, we also found that MetS significantly increased the risk of LREs. Therefore, the negative study did not change the finding.

The funnel plot was asymmetrical, and the $p$ values of Egger's test showed a publication bias in this meta analysis. However, through the trim and fill method, we obtained a new RR for LREs with MetS of 1.49 (95\% CI: $1.40-1.58, \mathrm{p}=0.000)$, and there was no obvious difference compared with our previous results. Therefore, we concluded that the results were robust, and MetS is associated with a moderately increased risk of LRE prevalence.

The results also indicated that preexisting MetS confers a statistically significant 1.56 - or 2.23-fold increased risk for LREs that is independent of other risk factors, an association also observed for its components. MetS can promote the occurrence of cardiovascular diseases, coronary heart disease and other diseases, and the degree of association between MetS and liver cancer is similar to the former [26, 27]. Nonalcoholic fatty liver disease (NAFLD), including non-alcoholic steatohepatitis (NASH), insulin resistance, and several metabolic abnormalities are closely related, thus suggesting a link between metabolic factors and cancer of the liver.

The findings from our study suggested a 1.33- or 2.33-fold increased risk of HCC development in patients with MetS. In addition, the study also 


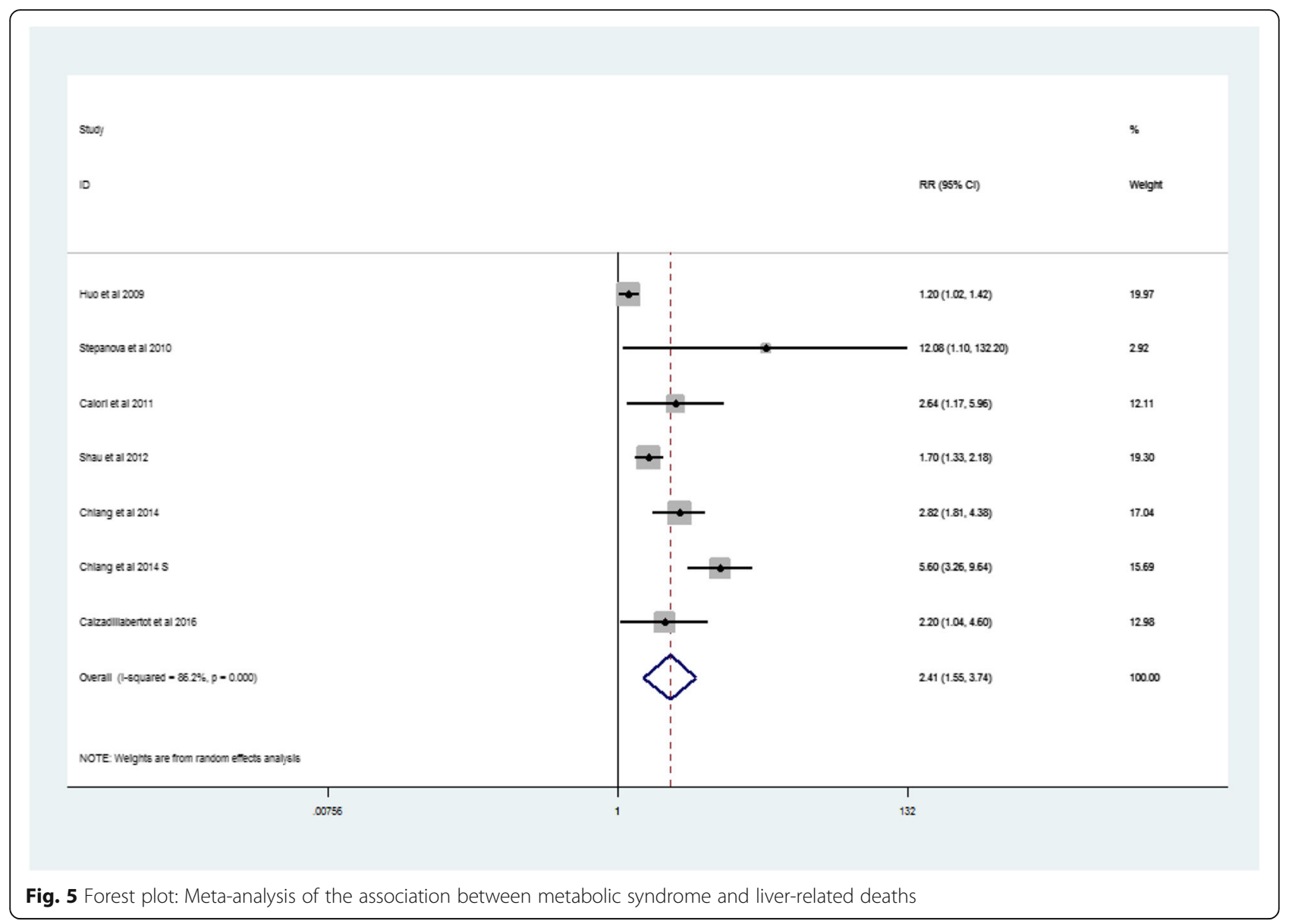

demonstrated a 1.55- or 3.74-fold increased risk of liver-related death in patients with MetS. Our results together indicate that MetS is associated with a moderately increased risk of LRE.

Analysis of related factors showed an overall 115\% increase in risk of LREs in HBV-positive cases (RR: 2.15, 95\% CI: 1.02-4.53). For HBV-negative cases, the probability indicated a $85 \%$ increase in risk (RR: 1.85 , 95\% CI: 1.53-2.24). This conclusion is consistent with viral hepatitis having been identified as a major risk factors for LREs [2, 3], because over time, patients infected with viral hepatitis develop cirrhosis and, eventually, HCC.

And there was some article showed the pooled RR for HCV subjects with IR was 1.89 (95\% CI, 1.542.33). It is because the presence of the $\mathrm{HCV}$ core protein will increase the level of tumor necrosis factor- $\alpha$, further leading to proteasomal degradation of the insulin receptor substrate, ultimately altering insulin function and promoting the development of IR. At the same time, IR further causes lipid accumulation in the liver and production of reactive oxygen species, and indirectly activates stellate cells, eventually leading to the occurrence of liver fibrosis [28].

The related factor analysis also indicated that non-Asian MetS populations are more likely than Asian populations to have LREs. There was a $73 \%$ increase in risk of LREs for Asians and an 112\% increase for non-Asians. The reason for this result is not yet clear, and more research is needed to provide an explanation. It is possible that the economy and the typical non-Western diet in Asia may explain the lower risk of MetS. And for Asian, the HBV vaccine is being recommended widespread. Meanwhile, the incidence of T2DM is lower in the Asian population, and it may have influenced the results. Some studies have reported that up to $90 \%$ of obese people in Europe have some degree of fatty degeneration in their liver and overweight increases the risk of HCC. The observed association between excess body weight and the increased risk of liver disease seems to support the reports that liver disease in obese individuals may be mediated through the development of NAFLD and NASH [12]. 


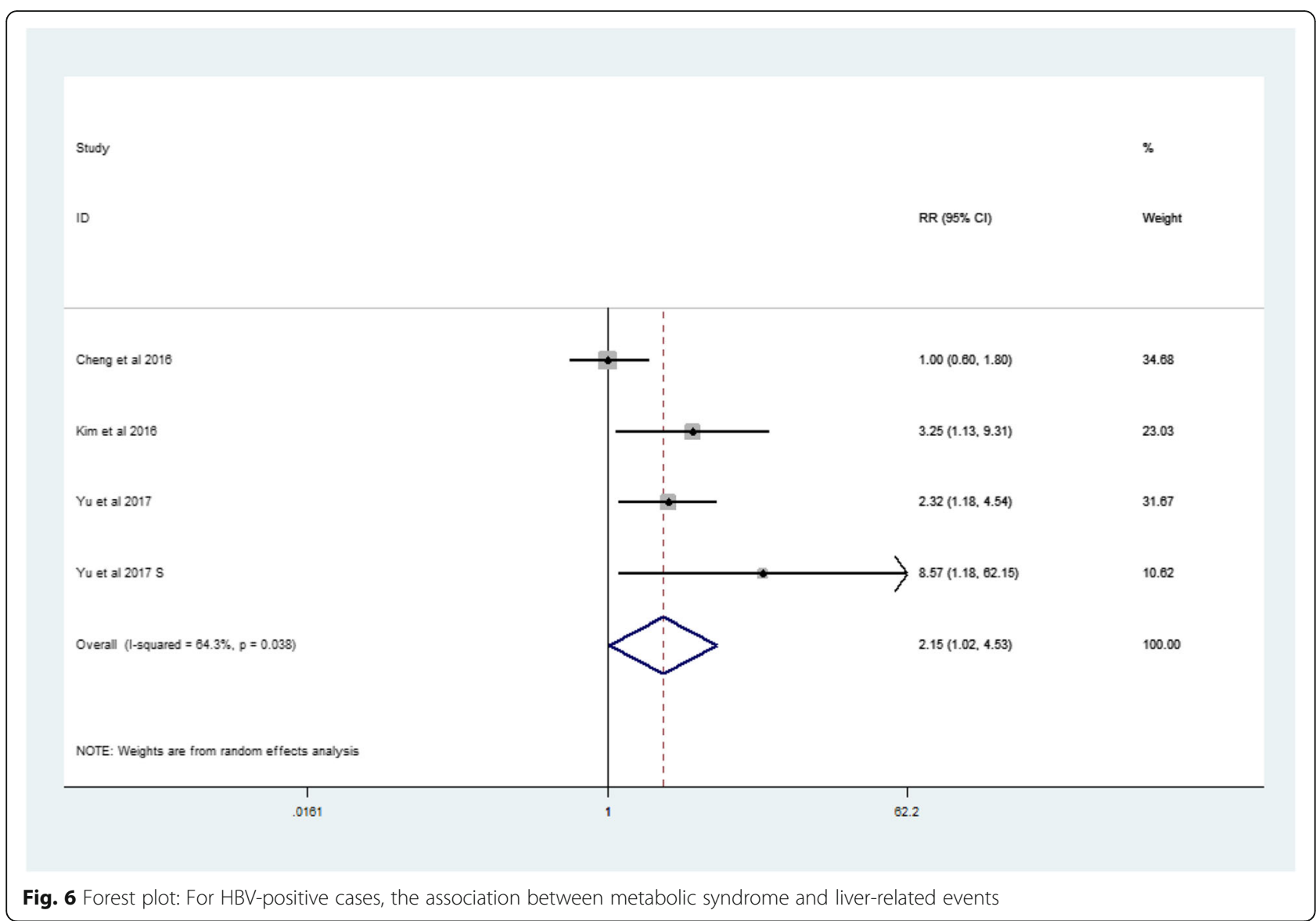

Our study suggests a relationship between MetS and LREs, but the mechanism that links the two is not fully understood. MetS is likely to be a proxy for other cancer risk factors, such as low physical activity, intake of high caloric food producing a high quantity of heat, high dietary fat intake, low fiber intake, and oxidative stress [29].The mechanisms by which DM induces $\mathrm{HCC}$ are related to insulin-like growth factor I (IGF-I) or insulin-like growth factor-binding protein-3 (IGFBP-3) [6]. The presence of insulin resistance and hepatic steatosis may support the hypothesis that diabetes promotes HCC, while the growth of insulin-like growth factor-1, the increase in leptin, the decrease in adiponectin and the imbalance of pro-inflammatory/anti-inflammatory cytokines are supported the appeal opinion [30]. There are several articles that study oxidative stress, cytokine effects, and other factors that contribute to the development of NAFLD. And because of the interaction between many metabolic factors, the study of their contribution to liver disease and liver cancer is more complicated. For example, deposition of free fatty acids and their metabolites in liver tissue is associated with hyperinsulinemia and insulin resistance, and they further promote hepatic steatosis [26].

Moreover, diabetic people have a higher risk of $\mathrm{HCV}$ infection, and diabetes is also associated with hyperinsulinemia. Both MetS and LREs are a problem worldwide, and growing evidence shows a relationship between MetS and an increased risk of LREs [31]. Insulin resistance and obesity are often thought to be linked to cancer, and diabetes is an independent prognostic factor for several common human malignancies, such as breast, colorectal, and prostate cancer $[32,33]$. Therefore, a link between MetS and cancer is also possible. Previous studies have shown that coexisting MetS in Chronic hepatitis B (CHB) patients increases the risk of different kinds of liver complications, such as liver fibrosis progression and liver cirrhosis [34]. The role of diabetes in liver cancer has been extensively studied, and a variety of biological mechanisms support an association [35-38]. For example, diabetes can affect the recurrence of liver cancer through hyperinsulinemia, hyperglycemia, or chronic inflammation.

A previous meta-analysis has described a relationship between hypertension and cancer, thus suggesting that 


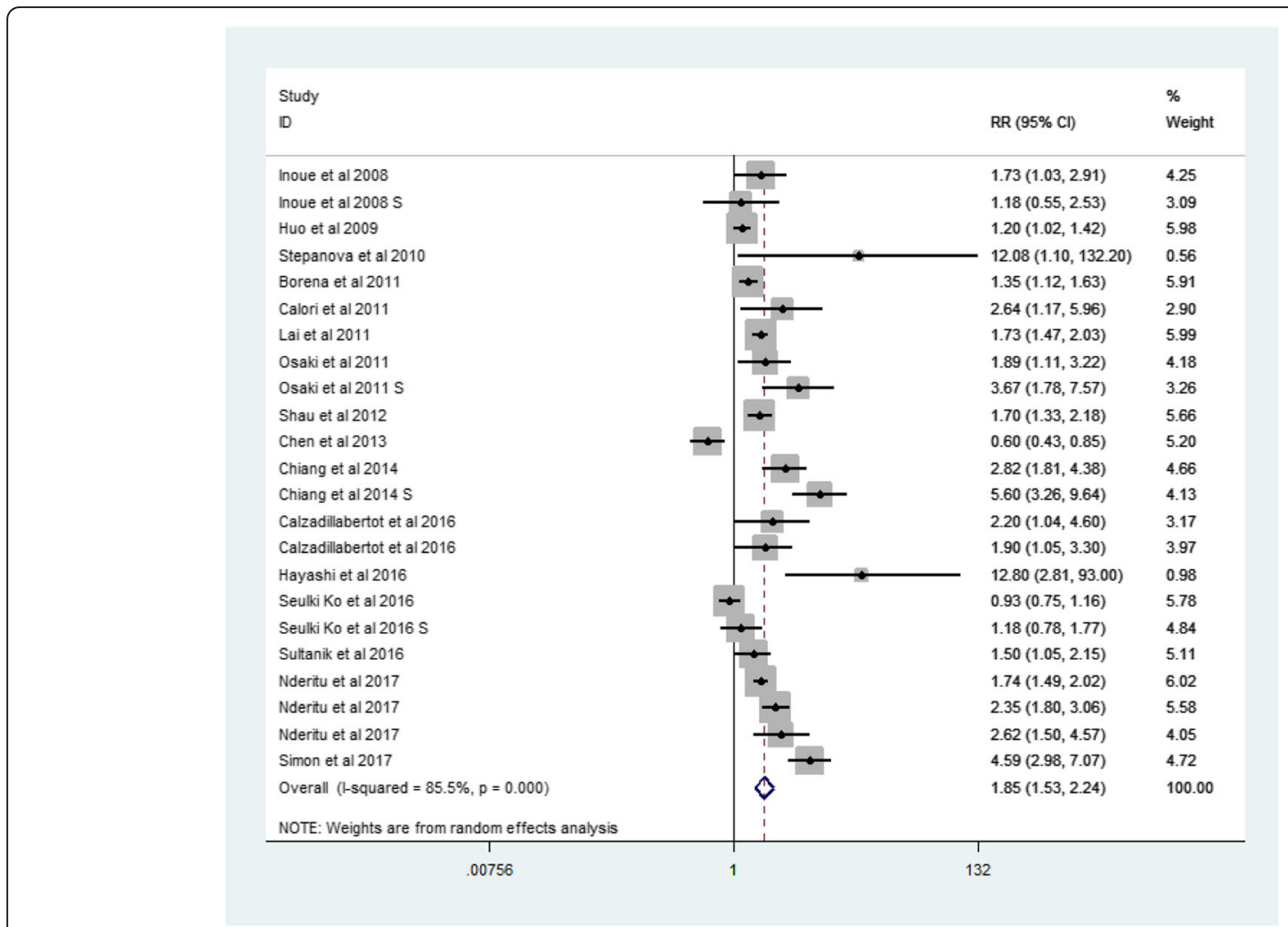

Fig. 7 Forest plot: For HBV-negative cases, the association between metabolic syndrome and liver-related events

Study
ID

Fig. 8 Forest plot: For Asian, the association between metabolic syndrome and liver-related events 


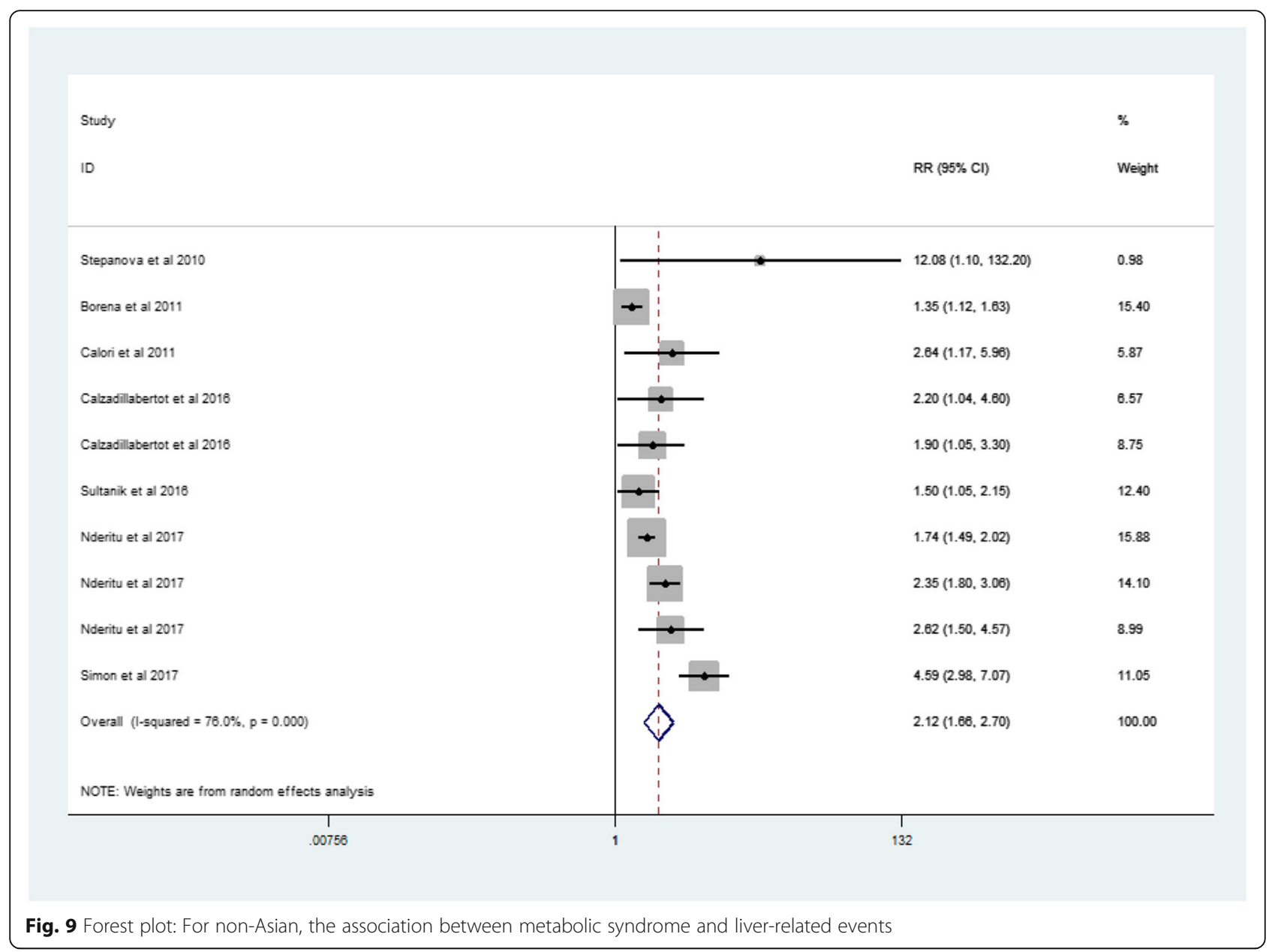

hypertension is associated with an increased risk of cancer death [26]. That study found that people with an average blood pressure of up to one in five have a cancer risk 2.8 times more than those with the lowest blood pressure. Another meta-analysis has examined the relationship between liver cancer and diabetes. Most studies have shown a significant increase in risk, with a combined risk ratio of 2.5 (95\% CI, 1.9-3.2) [39]. The above studies show that hypertension and diabetes as metabolic risk factors are significantly associated with the risk of liver disease, and decreasing the risk of MetS may also contribute to the long-term reduction of liver disease.

There are several limitations that should be considered when interpreting the findings of our meta-analysis. First, there are multiple factors that were not considered in the combined RR analysis, such as dietary habits, HCC family history, and other genetic risk factors, owing to the unavailability of these variables in the original study. Second, for diabetes and liver cancer risk meta-analysis, we referred data from observational studies, while those studies may have lower heterogeneity.
Third, because chronic liver disease can also cause diabetes, the link between diabetes and HCC may not be exact. Fourth, different controls on confounding factors also influenced the results. Finally, the meta-analysis was limited to English-language studies, which might have introduced publication bias.

However, our study has several strengths; for example, this article included more data than previous studies and examined links between MetS and cirrhosis, as well as liver-related deaths, among other factors. We also analyzed the geographic region and the population for hepatitis B and other parameters. The all cohort studies in our meta-analysis are of high quality and were able to detect potential associations and eliminate the possibility of recall and selection bias.

\section{Conclusion}

In conclusion, this population-based study showed that MetS is an important risk factor for the development of liver disease. Therefore, the control of the globally prevalent MetS may help reduce liver disease. At the 


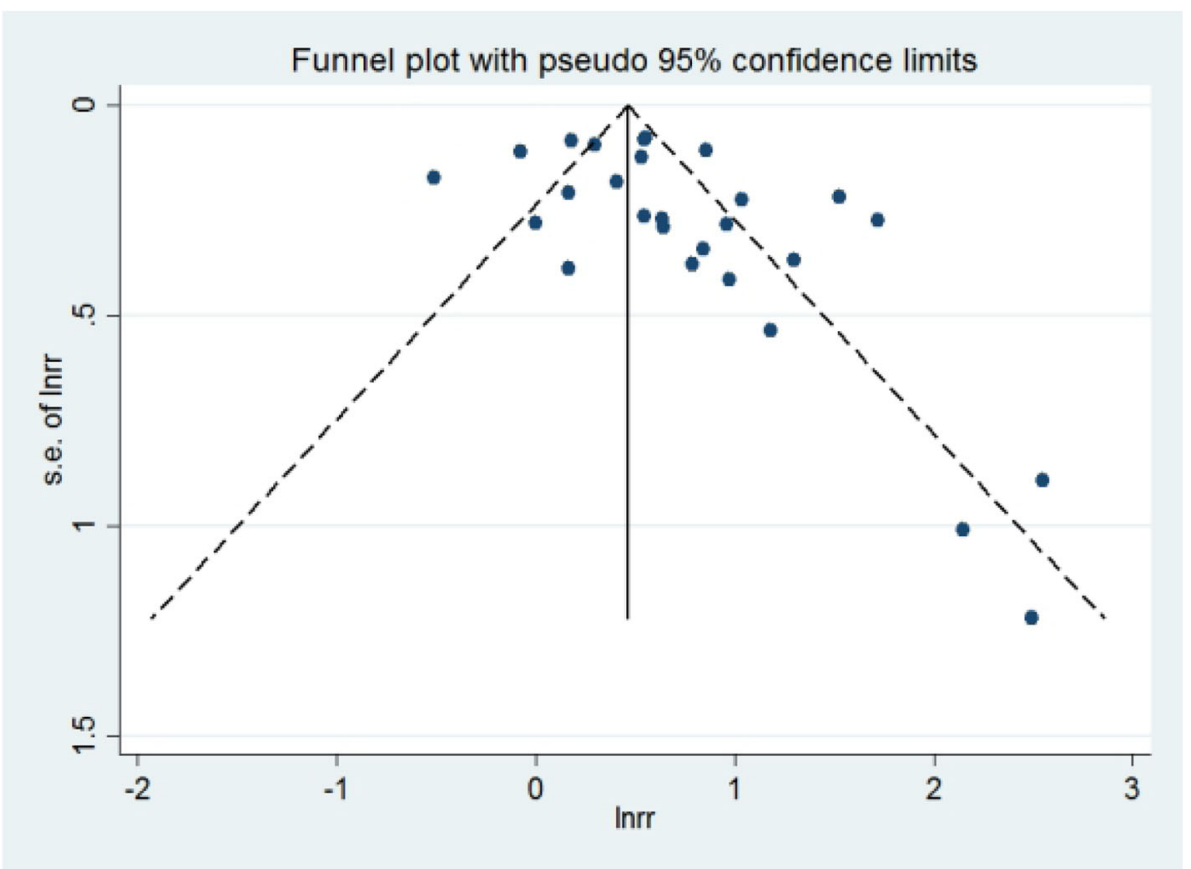

Fig. 10 Funnel plots for publication bias

same time, the metabolic syndrome and related factors are introduced in the paper. Trying to avoid these risk factors is a better treatment. More well-designed randomized controlled trials or prospective cohort or retrospective cohort studies are urgently needed to improve understanding of this risk.

\section{Abbreviations}

CHB: Chronic hepatitis B; Cl: Confidence interval; DM: Diabetes mellitus; HBV: Hepatitis B virus; HCC: Hepatocellular carcinoma; HCV: Hepatitis C virus; HR: Hazard ratio; IR: Insulin resistance; LREs: Liver-related events; MetS: Metabolic syndrome; NAFLD: Non-alcoholic fatty liver disease; NASH: Non-alcoholic steatohepatitis; NOS: Newcastle-Ottawa Scale; RR: Relative risk

\section{Acknowledgements}

-Not applicable.

\section{Funding}

-Not applicable.

\section{Availability of data and materials}

-All data generated or analysed during this study are included in this published article, and you can find these data in references $[1,6,9-25]$.

\section{Authors' contributions}

$-H R$, JW, YG and FY search the databases to find related articles, HR and WH analysis those articles and write the article. All authors read and approved the final manuscript.

\section{Ethics approval and consent to participate}

-Not applicable.

\section{Consent for publication}

-Not applicable.

\section{Competing interests}

-The authors declare that they have no competing interests.

\section{Publisher's Note}

Springer Nature remains neutral with regard to jurisdictional claims in published maps and institutional affiliations.

Received: 12 January 2019 Accepted: 8 April 2019

Published online: 25 April 2019

\section{References}

1. Cheng YK, Wong WS, Tse YK. Metabolic syndrome increases cardiovascular event but not hepatic event and death in patients with chronic hepatitis B. Hepatology. 2016;64(5):1507.

2. El-Serag HB. CURRENT CONCEPTS Hepatocellular Carcinoma. N Engl J Med. 2011;365(12):1118-27.

3. Siegel $A B$, Zhu AX. Metabolic syndrome and hepatocellular carcinoma: two growing epidemics with a potential link. Cancer. 2009;115(24):5651-61.

4. Byrne CD, Olufadi R, Bruce KD. Metabolic disturbances in non-alcoholic fatty liver disease. Clin Sci. 2009;116(7):539-64.

5. Esposito K, Chiodini P, Colao A. Metabolic syndrome and risk of Cancer: a systematic review and meta-analysis. Diabetes Care. 2012a;35(11):2402.

6. Chen $\mathrm{CT}$, Chen JY, Wang JH. Diabetes mellitus, metabolic syndrome and obesity are not significant risk factors for hepatocellular carcinoma in an HBV- and HCV-endemic area of southern Taiwan. Kaohsiung J Med Sci. 2013:29(8):451-9.

7. Egger M, Smith GD, Schneider M, Minder C. Bias in meta-analysis detected by a simple, graphical test. Bmj British Medical Journal. 1997;315(7109):629-34.

8. Begg CB, Mazumdar M. Operating characteristics of a rank correlation test for publication bias. Biometrics. 1994;50(4):1088.

9. Inoue M, Noda M, Kurahashi N. Impact of metabolic factors on subsequent cancer risk: results from a large-scale population-based cohort study in Japan. Eur J Cancer Prev. 2009;18(3):240-7.

10. Huo Tl, Hsu CY, Huang YH. Diabetes mellitus as an independent prognostic predictor and its association with renal dysfunction in patients with hepatocellular carcinoma. Liver Int. 2010;30(2):198-207.

11. Stepanova M, Rafiq N, Younossi ZM. Components of metabolic syndrome are independent predictors of mortality in patients with chronic liver disease: a population-based study. Gut. 2010:59(10):1410-5.

12. Borena W, Strohmaier S, Lukanova A. Metabolic risk factors and primary liver cancer in a prospective study of 578,700 adults. Int J Cancer. 2012;131(1): 193-200. 
13. Calori G, Lattuada G, Ragogna F. Fatty liver index and mortality: the Cremona study in the 15th year of follow-up. Hepatology. 2011;54(1):14552.

14. Lai SW, Chen PC, Liao KF. Risk of hepatocellular carcinoma in diabetic patients and risk reduction associated with anti-diabetic therapy: a population-based cohort study. Am J Gastroenterol. 2012;107(1):46.

15. Osaki Y, Taniguchi SI, Tahara A. Metabolic syndrome and incidence of liver and breast cancers in Japan. Cancer Epidemiol. 2012;36(2):141.

16. Shau WY, Shao YY, Yeh YC. Diabetes mellitus is associated with increased mortality in patients receiving curative therapy for hepatocellular carcinoma. Oncologist. 2012;17(6):856-62.

17. Chiang $\mathrm{CH}$, Lee LT, Hung SH. Opposite association between diabetes, dyslipidemia, and hepatocellular carcinoma mortality in the middle-aged and elderly. Hepatology. 2014;59(6):2207-15.

18. Calzadillabertot L, Vilargomez E, Torresgonzalez A. Impaired glucose metabolism increases risk of hepatic decompensation and death in patients with compensated hepatitis C virus-related cirrhosis. Digestive \& Liver Disease Official Journal of the Italian Society of Gastroenterology \& the Italian Association for the Study of the Liver. 2015;48(3):283-90.

19. Hayashi T, Ogawa E, Furusyo N. Influence of insulin resistance on the development of hepatocellular carcinoma after antiviral treatment for noncirrhotic patients with chronic hepatitis C. Infectious Agents \& Cancer 2016; 11(1):1-10.

20. Kim JH, Sinn DH, Gwak GY. Insulin resistance assessment is useful in risk stratification of hepatocellular carcinoma in chronic hepatitis B patients. Journal of Gastroenterology \& Hepatology. 2016;32(5).

21. Ko S, Yoon SJ, Kim D. Metabolic risk profile and Cancer in Korean men and women. Journal of Preventive Medicine \& Public Health. 2016;49(3):143-52.

22. Sultanik $P$, Kramer $L$, Soudan D. The relationship between liver stiffness measurement and outcome in patients with chronic hepatitis $C$ and cirrhosis: a retrospective longitudinal hospital study. Aliment Pharmacol Ther. 2016:44(5):505-13.

23. Nderitu P, Bosco C, Garmo H. The association between individual metabolic syndrome components, primary liver cancer and cirrhosis: a study in the Swedish AMORIS cohort. Int J Cancer. 2017.

24. Simon TG, King LY, Chong DQ. Diabetes, metabolic comorbidities and risk of hepatocellular carcinoma: results from two prospective cohort studies. Hepatology. 2017.

25. Yu MW, Lin CL, Liu CJ. Influence of metabolic risk factors on risk of hepatocellular carcinoma and liver-related death in men with chronic hepatitis B: a large cohort study. Gastroenterology. 2017;153(4):1006.

26. Welzel TM, Graubard Bl, Zeuzem S. Metabolic syndrome increases the risk of primary liver cancer in the United States: a study in the SEER-medicare database. J Hepatol. 2011;54(2):463-71.

27. Ratziu V, Bellentani S, Cortez-Pinto H. A position statement on NAFLD/NASH based on the EASL 2009 special conference. J Hepatol. 2010;53(2):372.

28. Patel $S$, Jinjuvadia $R$, Patel $R$, et al. Insulin resistance is associated with significant liver fibrosis in chronic hepatitis $C$ patients: a systemic review and Meta-analysis.[J]. J Clin Gastroenterol. 2015;50(1):80.

29. Alberti KGMM, Eckel RH, Grundy SM. Harmonizing the metabolic syndrome. Circulation. 2009;120(16):1640-5.

30. Esposito K, Chiodini P, Colao A, et al. Metabolic syndrome and risk of Cancer: a systematic review and meta-analysis[J]. Diabetes Care. 2012b; 35(11):2402

31. Wang C, Wang X, Gong G. Increased risk of hepatocellular carcinoma in patients with diabetes mellitus: a systematic review and meta-analysis of cohort studies. Int J Cancer. 2012;130(7):1639-48.

32. Yuhara H, Steinmaus $\mathrm{C}$, Cohen SE. Is diabetes mellitus an independent risk factor for Colon Cancer and rectal Cancer|[quest]. Am J Gastroenterol. 2011; 106(11):1911.

33. Peairs KS, Barone BB, Snyder CF. Diabetes mellitus and breast Cancer outcomes: a systematic review and Meta-analysis. Journal of Clinical Oncology Official Journal of the American Society of Clinical Oncology. 2011;29(1):40-6.

34. Wong GL, Wong WW. Commentary: coincidental metabolic syndrome increases the risk of liver fibrosis progression in patients with chronic hepatitis B - authors' reply. Aliment Pharmacol Ther. 2014;39(10):1236.

35. Wang YG, Wang P, Wang B. Diabetes mellitus and poorer prognosis in hepatocellular carcinoma: a systematic review and meta-analysis. PLoS One. 2014:9(5):e95485.
36. Baffy G. Editorial: hepatocellular carcinoma in type 2 diabetes: more than meets the eye. Am J Gastroenterol. 2012;107(1):53-5.

37. Salmon D, Bani-Sadr F, Loko MA. Insulin resistance is associated with a higher risk of hepatocellular carcinoma in cirrhotic HIV/HCV-co-infected patients: Results from ANRS CO13 HEPAVIH. J Hepatol. 2012;56(4):862-8.

38. Seshasai SR, Kaptoge $S$, Thompson A. Diabetes mellitus, fasting glucose, and risk of cause-specific death. N Engl J Med. 2011;364(9):829.

39. HB E-S, Hampel H, Javadi F. The association between diabetes and hepatocellular carcinoma: a systematic review of epidemiologic evidence. Clinical gastroenterology and hepatology : the official clinical practice. journal of the American Gastroenterological Association. 2006:4(3):369-80.

\section{Ready to submit your research? Choose BMC and benefit from:}

- fast, convenient online submission

- thorough peer review by experienced researchers in your field

- rapid publication on acceptance

- support for research data, including large and complex data types

- gold Open Access which fosters wider collaboration and increased citations

- maximum visibility for your research: over $100 \mathrm{M}$ website views per year

At $\mathrm{BMC}$, research is always in progress.

Learn more biomedcentral.com/submissions 